\title{
Development of a Phage Cocktail to Control Proteus mirabilis Catheter-associated Urinary Tract Infections
}

\author{
Luís D. R. Melo ${ }^{1}$, Patrícia Veiga ${ }^{1}$, Nuno Cerca ${ }^{1}$, Andrew M. Kropinski ${ }^{2}$, Carina Almeida ${ }^{1}$, \\ Joana Azeredo ${ }^{1}$ and Sanna Sillankorva ${ }^{1 *}$ \\ 1 Laboratório de Investigação em Biofilmes Rosário Oliveira, Centre of Biological Engineering, University of Minho Braga, \\ Braga, Portugal, ${ }^{2}$ Departments of Food Science, Molecular and Cellular Biology, and Pathobiology, University of Guelph, \\ Guelph, ON, Canada
}

Proteus mirabilis is an enterobacterium that causes catheter-associated urinary tract infections (CAUTIs) due to its ability to colonize and form crystalline biofilms on the catheters surface. CAUTIs are very difficult to treat, since biofilm structures are highly tolerant to antibiotics. Phages have been used widely to control a diversity of bacterial species, however, a limited number of phages for $P$. mirabilis have been isolated and studied. Here we report the isolation of two novel virulent phages, the podovirus vB_PmiP_5460 and the myovirus vB_PmiM_5461, which are able to target, respectively, 16 of the 26 and all the Proteus strains tested in this study. Both phages have been characterized thoroughly and sequencing data revealed no traces of genes associated with lysogeny. To further evaluate the phages' ability to prevent catheter's colonization by Proteus, the phages adherence to silicone surfaces was assessed. Further tests in phage-coated catheters using a dynamic biofilm model simulating CAUTIs, have shown a significant reduction of $P$. mirabilis biofilm formation up to $168 \mathrm{~h}$ of catheterization. These results highlight the potential usefulness of the two isolated phages for the prevention of surface colonization by this bacterium.

Keywords: bacteriophages, bacteriophage therapy, biofilms, urinary tract infection, Proteus mirabilis, phage cocktail

\section{INTRODUCTION}

Indwelling urinary catheters are medical devices used by millions of people to relieve urinary retention and urinary incontinence. It has been estimated that more than 100 million urethral catheters are fitted each year in a range of healthcare facilities (Getliffe and Newton, 2006). Despite the important benefits brought by the use of these devices, catheters provide a suitable surface for the colonization of microorganisms and may further place at risk the patients' health, due to infections. In USA, catheter-associated urinary tract infections (CAUTIs) account for up to $40 \%$ of hospital-acquired infections (Saint et al., 2008). Also, 70\% of urinary tract infections (UTIs) are associated with urinary catheters (Burton et al., 2011; Weber et al., 2011) and approximately 20\% patients will suffer a catheterization during their hospital stay, especially in intensive care units (Saint and Lipsky, 1999). 
The duration of the catheterization is a crucial risk factor of CAUTI development as almost all long-term catheterized (>28 days) patients develop a CAUTI, whereas in short-term ( $<7$ days) catheterized patients only $10-50 \%$ develop an infection (Morris et al., 1999).

Proteus mirabilis is a leading cause of CAUTIs, being associated with up to $44 \%$ of all CAUTIs in the USA (O'Hara et al., 2000; Jacobsen et al., 2008). This microorganism, isolated from soil, stagnant water, sewage, and human intestinal tract, is associated with complicated infections, long-term catheterizations and urinary stone (struvite) formation (Armbruster and Mobley, 2012). P. mirabilis is a dimorphic bacterium that expresses thousands of flagella responsible for its swarming ability (Hoeniger, 1965). The virulence factors of $P$. mirabilis that contribute to the establishment of CAUTIs include the expression of fimbriae, that mediate the attachment to host cells and to catheters, and the consequent formation of dense biofilms on catheter surfaces (Armbruster and Mobley, 2012). Furthermore, it produces urease which is responsible for urea hydrolysis to carbon dioxide and ammonia that raises urine pH to above 8.3 (Stickler et al., 1998; Broomfield et al., 2009).

The microorganisms that colonize indwelling urinary catheters are commonly associated with antibiotic resistance and, thus, biofilm structures are frequently reported as reservoirs of antibiotic-resistant bacteria (Chenoweth et al., 2014). Although antibiotic therapy is successful in the majority of the cases, there has been a dramatic increase on antibiotic resistance among CAUTI-causing bacteria, including P. mirabilis (Wang et al., 2014). This fact makes it difficult to treat CAUTIs, highlighting the need for alternative preventive measures. During the last decades, the use of virulent bacteriophages (or phages) has re-emerged for therapeutic purposes (Viertel et al., 2014). After replicating inside the bacterial host, they cause cell lysis and release of phage progeny, which are able to infect neighboring cells. Phages are potential specific antibacterial agents, as they have self-replicating nature in the presence of the host cells, being eliminated from the human body in their absence (Azeredo and Sutherland, 2008). Furthermore, they are active against antibiotic-resistant bacteria, and phage preparations containing several phages, also known as phage cocktails, can be developed to increase their activity spectrum (Gu et al., 2012).

In the present study, two $P$. mirabilis-specific phages were isolated and characterized, and their effectiveness to control biofilm formation on silicone catheters was assessed in a dynamic biofilm model simulating CAUTIs.

\section{MATERIALS AND METHODS}

\section{Bacterial Strains and Culture Conditions}

A total of 26 Proteus spp. (18 P. mirabilis) strains were used in this study. Additionally, other members of the Enterobacteriaceae were used to assess the lytic spectra of the phages. The complete list of strains used in this study is provided in Table 1. Reference strains were obtained from Salmonella Genetic Stock Centre (SGSC) and Colección Española de Cultivos Tipo (CECT), while isolates were obtained from Laboratório de Análises Clínicas
S. Lázaro (Braga, Portugal), from urine samples. Species was identified using selective agar media and biochemical tests. Bacteria were grown at $37^{\circ} \mathrm{C}$ in Tryptic Soy Broth (TSB; Liofilchem), on Tryptic Soy Agar (TSA; 1.5\% agar) or in Artificial Urine (AU) (Brooks and Keevil, 1997) supplemented with 0.3\% glucose (Silva et al., 2010).

\section{Phage Isolation, Production, and Titration}

Bacteriophage isolation was performed using the enrichment procedure, essentially as described before (Melo et al., 2014b), using raw sewage (Braga). Briefly, $50 \mathrm{~mL}$ of centrifuged effluent was mixed with the same volume of double-strength TSB and then inoculated with thirteen $P$. mirabilis strains (labeled with an "a" in Table 1). Fifty micro liter of each exponentially grown $P$. mirabilis culture were used. This solution was incubated for $18 \mathrm{~h}$ at $37^{\circ} \mathrm{C}, 120 \mathrm{rpm}$, centrifuged $\left(10 \mathrm{~min}, 10,000 \times g, 4^{\circ} \mathrm{C}\right)$ and the supernatant filtered through a $0.22 \mu \mathrm{m}$ polyethersulfone membrane (GVS - Filter Technology). The presence of phages was checked by performing spot assays on bacterial lawns. Inhibition zones were purified to isolate all different phages on the respective bacterial host. Plaque picking was repeated until single-plaque morphology was observed and ten plaques of each isolated phage were measured and characterized.

Phage particles were produced using the plate lysis and elution method as described previously (Sambrook and Russell, 2001) with some modifications. Briefly, $10 \mu \mathrm{L}$ phage suspension was spread on host bacterial lawns using a paper strip and incubated for $14-16 \mathrm{~h}$ at $37^{\circ} \mathrm{C}$. After, $3 \mathrm{~mL}$ of SM Buffer [ $100 \mathrm{mM} \mathrm{NaCl}$, $8 \mathrm{mM} \mathrm{MgSO}_{4}, 50 \mathrm{mM}$ Tris/HCl ( $\mathrm{pH} 7.5$ ), 0.002\% (w/v) gelatin] were added to each plate and incubated for $8 \mathrm{~h}(120 \mathrm{rpm}$ on a PSU-10i Orbital Shaker (BIOSAN), $\left.4^{\circ} \mathrm{C}\right)$. Subsequently, the liquid and top-agar were collected, centrifuged $(10 \mathrm{~min}$, $\left.10,000 \times g, 4^{\circ} \mathrm{C}\right)$. The lysate was further concentrated with PEG 8000 and then purified with chloroform and stored at $4^{\circ} \mathrm{C}$.

Phage titration was performed according to the double agar overlay technique (Kropinski et al., 2009). Briefly, $100 \mu l$ of diluted phage solution, $100 \mu \mathrm{l}$ of host bacteria culture, and $3 \mathrm{~mL}$ of soft agar were poured onto a Petri plate containing a thin layer of TSA. After overnight incubation at $37^{\circ} \mathrm{C}$, the plaque forming units (PFUs) were determined.

\section{Lytic Spectra of the Isolated Phages}

The host range of the two isolated phages was determined by pipetting $10 \mu \mathrm{l}$ of diluted phage solution $\left(10^{8}\right.$ PFU.mL $\left.{ }^{-1}\right)$ on lawns of indicated bacterial strains (Table 1). Plates were incubated $12 \mathrm{~h}$ at $37^{\circ} \mathrm{C}$, and the presence and absence of inhibition zones indicating host sensitivity were reported.

\section{Electron Microscopy}

The morphology of phage particles was observed by transmission electron microscopy, as previously described (Melo et al., 2014b). Briefly, phage particles were collected after centrifugation $\left(1 \mathrm{~h}, 25,000 \times g, 4^{\circ} \mathrm{C}\right)$. The pellet was washed twice in tap water using the same centrifugation conditions. Phages were further deposited on copper grids with carbon-coated Formvar 
TABLE 1 | Phages lytic spectra on the bacterial strains used in this study.

\begin{tabular}{|c|c|c|c|c|}
\hline Species & Strain & Source & Infectivity Pm5460 & Infectivity Pm5461 \\
\hline \multirow[t]{18}{*}{ Proteus mirabilis } & ATCC $29906^{a}$ & Culture collection & + & + \\
\hline & ATCC $14153^{a}$ & Culture collection & + & + \\
\hline & SGSC $5460^{a}$ & Culture collection & + & + \\
\hline & SGSC $5461^{a}$ & Culture collection & - & + \\
\hline & $933^{a}$ & Human urine isolate (Braga) & - & + \\
\hline & SGSC $3360^{a}$ & Culture collection & - & + \\
\hline & SGSC $5446^{a}$ & Culture collection & + & + \\
\hline & SGSC $5447^{a}$ & Culture collection & + & + \\
\hline & SGSC $5445^{\mathrm{a}}$ & Culture collection & - & + \\
\hline & SGSC 5448a & Culture collection & + & + \\
\hline & SGSC 5449a & Culture collection & + & + \\
\hline & SGSC $5450^{a}$ & Culture collection & - & + \\
\hline & 2380 & Human urine isolate (Braga) & + & + \\
\hline & 2388B & Human urine isolate (Braga) & + & + \\
\hline & 9229 & Human urine isolate (Braga) & - & + \\
\hline & SLaz1 ${ }^{a}$ & Human urine isolate (Braga) & + & + \\
\hline & SLaz2 & Human urine isolate (Braga) & + & + \\
\hline & SLaz3 & Human urine isolate (Braga) & + & + \\
\hline \multirow[t]{6}{*}{ Proteus vulgaris } & ATCC 6380 & Culture collection & - & + \\
\hline & ATCC 6896 & Culture collection & - & + \\
\hline & ATCC 13315 & Culture collection & + & + \\
\hline & ATCC 29905 & Culture collection & - & + \\
\hline & SGSC 3359 & Culture collection & + & + \\
\hline & SGSC 5469 & Culture collection & + & + \\
\hline Proteus hauseri & ATCC 13315 & Culture collection & - & + \\
\hline Proteus penneri & ATCC 33519 & Culture collection & + & + \\
\hline Citrobacter freundii & SGSC 5345 & Culture collection & - & - \\
\hline Citrobacter koseri & CK18 & Human pus isolate (Braga) & - & - \\
\hline Cronobacter sakazakii & ATCC 29544 & Culture collection & - & - \\
\hline Enterobacter aerogenes & ATCC 13048 & Culture collection & - & - \\
\hline \multirow[t]{5}{*}{ Escherichia coli } & ATCC 11775 & Culture collection & - & - \\
\hline & CECT 434 & Culture collection & - & - \\
\hline & Ec7 & Human urine isolate (Braga) & - & - \\
\hline & Ec8 & Human urine isolate (Braga) & - & - \\
\hline & Ec9 & Human urine isolate (Braga) & - & - \\
\hline Escherichia hermannii & ATCC 33650 & Culture collection & - & - \\
\hline Klebsiella pneumoniae & ATCC 11296 & Culture collection & - & - \\
\hline \multirow[t]{2}{*}{ Morganella morganii } & SGSC 5703 & Culture collection & - & - \\
\hline & M12 & Human urine isolate (Braga) & - & - \\
\hline Providencia rettgeri & $\mathrm{R} 1$ & Human sputum isolate (Braga) & - & - \\
\hline Providencia stuartii & S7 & Human urine isolate (Braga) & - & - \\
\hline Salmonella Enteritidis & ATCC 13076 & Culture collection & - & - \\
\hline Salmonella Typhimurium & ATCC 43971 & Culture collection & - & - \\
\hline
\end{tabular}

${ }^{a}$ strain used on the enrichment procedure for phage isolation.

films, stained with $2 \%$ uranyl acetate ( $\mathrm{pH} 4.0)$. Phages were observed using a Philips EM 300 electron microscope, and magnification was monitored with T4 phage tails (Ackermann, 2009).

\section{One-step Growth Curve}

One-step growth curve studies were performed as described previously (Rahman et al., 2011), with some modifications. Briefly, $10 \mathrm{~mL}$ mid-exponential-phase culture, $\mathrm{OD}_{620} 0.5$, was harvested by centrifugation $\left(5 \mathrm{~min}, 7000 \times \mathrm{g}, 4^{\circ} \mathrm{C}\right)$ and resuspended in $5 \mathrm{~mL}$ fresh TSB medium in order to obtain an $\mathrm{OD}_{620}$ of 1.0. The same volume of phage solution was added in order to achieve a multiplicity of infection (MOI) of 0.005 . After adsorption during $5 \mathrm{~min}\left(37^{\circ} \mathrm{C}, 120 \mathrm{rpm}\right)$ the mixture was centrifuged as described above, and the pellet resuspended in $10 \mathrm{~mL}$ of fresh TSB. Samples were taken every $5 \mathrm{~min}$ over a period of $30 \mathrm{~min}$ and every $10 \mathrm{~min}$ until $1 \mathrm{~h}$ of infection. 


\section{DNA Isolation, Genome Sequencing, and Annotation}

Phage DNA was extracted as described before (Melo et al., 2014a). Purified phages were treated with $0.016 \%(\mathrm{v} / \mathrm{v}) \mathrm{L} 1$ buffer [300 mM NaCl, $100 \mathrm{mM}$ Tris/HCl (pH 7.5), $10 \mathrm{mM}$ EDTA, $0.2 \mathrm{mg}$ BSA $\mathrm{mL}^{-1}, 20 \mathrm{mg}$ RNase $\mathrm{A} \mathrm{mL}^{-1}$ (Sigma), $6 \mathrm{mg}$ DNase $\mathrm{I} \mathrm{mL}^{-1}$ (Sigma)] for $2 \mathrm{~h}$ at $37^{\circ} \mathrm{C}$. After a thermal inactivation of the enzymes for $30 \mathrm{~min}$ at $65^{\circ} \mathrm{C}$, $50 \mu \mathrm{g}$ proteinase $\mathrm{K} \mathrm{ml}^{-1}, 20 \mathrm{mM}$ EDTA, and 1\% SDS were added and proteins were digested for $18 \mathrm{~h}$ at $56^{\circ} \mathrm{C}$. This was followed by phenol:chloroform:isoamyl alcohol solution (25:24:1, $\mathrm{v} / \mathrm{v}$ ) and chloroform extractions. DNA was precipitated with isopropanol and $3 \mathrm{M}$ sodium acetate ( $\mathrm{pH} 4.6)$, centrifuged (15 min, $7,600 \times g, 4^{\circ} \mathrm{C}$ ), and the pellet air-dried and further resuspended in nuclease-free water (Cleaver Scientific). Genome sequencing was performed on a 454 sequencing platform (Plate-forme d' Analyses Génomiques at Laval University, Québec city, QC, Canada) to 50-fold coverage. Sequence data was assembled using SeqMan NGen4 software (DNASTAR, Madison, WI, USA). Phage genomes were autoannotated, using MyRAST (Aziz et al., 2008) and the presence of nonannotated CDSs, along with genes in which the initiation codon was miscalled, were checked manually using Geneious 6.1.6 (Biomatters). Potential frameshifts were checked with BLASTX (Altschul et al., 1997) and BLASTP was used to check for homologous proteins (Altschul et al., 1990), with an $\mathrm{E}$ value threshold of $<1 \times 10^{-5}$ and at least $80 \%$ query. Pfam (Finn et al., 2014) was used for protein motif search, with the same cutoff parameters as used with BLASTP. Protein parameters (molecular weight and isoelectric point) were determined using ExPASy Compute pI/Mw (Wilkins et al., 1999). The presence of transmembrane domains was checked using TMHMM (Kall and Sonnhammer, 2002) and Phobius (Kall et al., 2004), and membrane proteins were annotated when both tools were in concordance. The search of tRNA encoding genes was performed using ARAGORN (Laslett and Canback, 2004) and tRNAscan-SE (Schattner et al., 2005). Fragments $100 \mathrm{bp}$ upstream of each predicted ORF were extracted and MEME (Bailey et al., 2009) was used to search for putative promoter regions that were further manually verified. For the same purposes, PHIRE was also used (Lavigne et al., 2004). ARNold (Naville et al., 2011) was used to predict rhoindependent terminators and the energy was calculated using Mfold (Zuker, 2003). EMBOSS Stretcher (Rice et al., 2000) and CoreGenes (Zafar et al., 2002) were used for whole genome comparisons between $P$. mirabilis phages and their closest relatives. Phage 5460 was compared with Enterobacteria phages: K1-5 (NC_008152), UAB_Phi78 (NC_020414), and SP6 (AY370673). Phage 5461 comparisons were performed with Yersinia phage phiR1-RT (HE956709), and Salmonella phages STP4-a (KJ000058) and S16 (HQ331142). For phylogenetic analysis, homologous proteins were identified using BLASTP against the virus database at NCBI. A phylogenetic tree was constructed using "One Click" phylogeny. $\mathrm{fr}^{1}$ (Dereeper et al.,

${ }^{1}$ http://phylogeny.lirmm.fr/phylo_cgi/simple_phylogeny.cgi
2008). The data was exported in Newick tree format and opened in FigTree ${ }^{2}$.

\section{Evaluation of the Degree and Stability of Phage Coating}

Silicone coupons were placed in 24 -well plates and $1.7 \mathrm{~mL}$ of both phage solutions with different concentrations $\left(10^{6}, 10^{7}, 10^{8}\right.$, $10^{9}$, and $10^{10}$ PFU. $\mathrm{mL}^{-1}$ ) were added. Plates were incubated overnight at room temperature without agitation. Coupons were removed, washed with SM Buffer and placed in a new well with the same volume SM Buffer.

Different sonication conditions were optimized to assure the efficient removal of viral particles without compromising the particles viability. Accordingly, a loss of viability for both phages was observed with an increase of the sonication period (>5 s) and amplitude (loss of viability at $25 \%>$ loss of viability at $22 \%$ ) (Supplementary Figure S1). While it seems that amplitude increase has a negative effect on phage viability, there were no statistical differences between the 22 and $25 \%$ of amplitude $(p>0.05)$. Statistical differences were only noticed between the different sonication times for phage 5461 (for both amplitudes) and for phage 5460 (only for 25\% of amplitude) ( $p<0.05$ ). Consequently, coupons were further sonicated for $5 \mathrm{sec}$ at $22 \%$ amplitude and PFU.mL $\mathrm{mL}^{-1}$ were determined. The sonication was optimized to assure the efficient removal of viral particles without compromising virus viability using two different amplitudes (22 and 25\%, Sonics Vibra-Cell VC 505 - VC 750 sonicator) during different sonication times $(5,10$, and $20 \mathrm{~s})$.

To further evaluate the "natural" release of phages from the silicone surface overtime, phage-coated coupons, were exposed to AU supplemented with $0.3 \%$ glucose at $37^{\circ} \mathrm{C}$. For the surface coating, the concentration of the initial suspension was adjusted to obtain a concentration of adhered phages ranging from $10^{5}$ to $10^{6}$ phages $/ \mathrm{cm}^{2}$. At specific time points $(2,4,6,24,48,72$, and $96 \mathrm{~h}$ ) the number of released phages was determined by plaque assay.

\section{Screening of $P$. mirabilis Strains for Biofilm Formation Ability}

The stability of the phages was assessed after incubating the phages on $\mathrm{AU}$ at $37^{\circ} \mathrm{C}$ for $168 \mathrm{~h}$ with phages samples titration every $24 \mathrm{~h}$.

For biofilm formation assays, 15 P. mirabilis strains (Table 1) were grown in $10 \mathrm{~mL}$ of $\mathrm{AU}$ supplemented with $0.3 \%$ glucose, and incubated for 16-18 h (orbital shaker ES-20/60 (BIOSAN), $120 \mathrm{rpm}, 37^{\circ} \mathrm{C}$ ). Bacterial cultures were centrifuged $(3 \mathrm{~min}$, $5,000 \times g)$ and pellets resuspended in fresh $\mathrm{AU}$ to an $\mathrm{OD}_{620 \mathrm{~nm}}$ of $0.1\left(\sim 1 \times 10^{8}\right.$ CFU.mL $\left.{ }^{-1}\right)$. Twenty $\mu$ l of each culture were added to $180 \mu \mathrm{l}$ of fresh AU in a 96-well plate $(n=6)$ and were incubated for $48 \mathrm{~h}$, at the conditions described above, with media renewal after $24 \mathrm{~h}$. Negative controls were performed with $200 \mu \mathrm{l}$ of $\mathrm{AU}(n=6)$.

Biofilm biomass was quantified as previously described (Pires et al., 2011), with some modifications. Briefly, after $48 \mathrm{~h}$ of

${ }^{2}$ http://tree.bio.ed.ac.uk/software/figtree/ 
incubation, all media was removed and the wells washed twice with phosphate buffered saline (PBS), $\mathrm{pH}$ 7.5. After PBS removal, $220 \mu \mathrm{l}$ of methanol were added for $20 \mathrm{~min}$, the methanol was removed, and the plates were air-dried. Then, $220 \mu \mathrm{L}$ of $1 \%$ crystal violet (w/v, Merck) were added to each well, incubated for $10 \mathrm{~min}$ at room temperature, and washed with tap water. Finally, $220 \mu \mathrm{L}$ of $33 \%$ acetic acid (v/v, Fisher) were added to each well to dissolve the stain and the absorbance measured at $570 \mathrm{~nm}$, in an ELISA reader (Tecan). Three independent experiments were performed. Strains were classified regarding biofilm formation, as previously described (Stepanovic et al., 2000). A cut-off $\left(\mathrm{OD}_{\mathrm{c}}\right)$ was defined with three standard deviations above negative controls (AU). Four classes were defined: $\mathrm{OD} \leq \mathrm{OD}_{\mathrm{c}}-$ nonbiofilm forming strain; $\mathrm{OD}_{\mathrm{c}}<\mathrm{OD} \leq 2 \times \mathrm{OD}_{\mathrm{c}}-$ weak biofilm forming strain; $2 \times \mathrm{OD}_{\mathrm{c}}<\mathrm{OD} \leq 4 \times \mathrm{OD}_{\mathrm{c}}-$ moderate biofilm forming strain; $4 \times \mathrm{OD}_{\mathrm{c}}<\mathrm{OD}-$ strong biofilm forming strain.

\section{Efficacy of a Phage Cocktail under Dynamic Biofilm Formation Conditions}

To mimic catheter conditions, biofilms were formed in a continuous model on Foley catheters (Silkemed Uro-cath 2Way, Overpharma). The flow was set to $0.5 \mathrm{~mL} \cdot \mathrm{min}^{-1}$ to mimic the actual average flow in a catheterized patient (Jones et al., 2005; Levering et al., 2014). With the exception of the catheter, all other tubing of the system was autoclaved $\left(15 \mathrm{~min}, 121^{\circ} \mathrm{C}\right)$. Under sterile conditions and with the aid of a sterile scalpel the two ends of the Foley catheters were removed to allow its attachment to the other tubing of the system. A phage cocktail containing the same concentration of phages Pm5460 and Pm5461 was prepared. Three $\mathrm{mL}$ of the phage cocktail at $10^{9} \mathrm{PFU}_{\mathrm{mL}}{ }^{-1}$ (to obtain a $10^{6}$ PFU.cm ${ }^{-2}$ coating) were added to each catheter, the catheter was sealed and phage binding to the catheter material allowed to occur overnight at room temperature under static conditions. On the following day, all non-bound phages were removed flowing the catheter with SM Buffer, the flow systems was mounted under sterile conditions, and then catheters were supplied with AU inoculated with $1 \times 10^{5}$ CFU.mL ${ }^{-1}$ of both P. mirabilis SGSC 5446 and SGSC 5449 (previously grown for $16 \mathrm{~h}$ in $10 \mathrm{~mL}$ AU supplemented with $0.3 \%$ glucose, centrifuged ( $3 \mathrm{~min}, 5,000 \times g$ ) and suspended at the desired concentration). Phage-coated and non-coated catheters were supplied with a continuous flow of fresh urine with the bacterial suspension for $24 \mathrm{~h}$, and after with $\mathrm{AU}$ for up to $168 \mathrm{~h}$ with no recirculation of the medium. At 48, 96, and $168 \mathrm{~h}, 2 \mathrm{~cm}$ of each catheter were removed: $1 \mathrm{~cm}$ for microscopy analysis, and $1 \mathrm{~cm}$ for CFU analysis. To determine the number of viable cells, samples were resuspended in $1.5 \mathrm{~mL} \mathrm{NaCl} 0.9 \%$, sonicated for $5 \mathrm{~s}$ at $22 \%$, and plated in TSA plates. Four independent assays were performed. The sonication step was previously optimized to assure complete detachment with no loss of cellular viability (see Supplementary Figure S1). Briefly, to optimize the sonication conditions for the detachment of cells, $1 \mathrm{~mL}$ of a fresh inoculum of $P$. mirabilis set to an $\mathrm{OD}_{620 \mathrm{~nm}}$ of 0.1 was added to each well of a 24-well plate and sonicated using two different amplitudes (22 and 25\%, Sonics Vibra-Cell VC 505 - VC 750 sonicator) during different sonication times $(5,10,15,20$, and 40 s). Viable cells at each time point and amplitude were determined. Three independent assays were performed. The best conditions were applied to biofilms samples, and efficient removal was confirmed by microscopy.

\section{Microscopy Observations of the Biofilms}

Epifluorescence observations: To assess the ability of phages to control the biofilms formed on silicone catheters, catheter sections were stained with DAPI. Briefly, $0.5 \mathrm{~cm}$ sections of phage-coated or non-coated catheters taken at each time points were stained with $100 \mu \mathrm{l}\left(100 \mu \mathrm{g} \cdot \mathrm{mL}^{-1}\right)$ of DAPI and placed in the dark for $10 \mathrm{~min}$. The excess of dye was removed using absorbent paper, the catheter sections were placed on microscope slides and analyzed using an epifluorescence microscope (Olympus, Model BX51, Hamburg, Germany) equipped with a CCD camera (Olympus, Model DP72) with a filter-block for DAPI fluorescence (Ex 365-370; Barrier 400; Em LP 421).

Scanning electron microscopy (SEM) observations: SEM observations were performed on washed (PBS) catheter sections, which had been gradually dehydrated in absolute ethanol (Merck) solutions ( 15 min each in 10, 25, 40, 50, 70, 80, and 100\% v/v). The catheter sections, kept in a desiccator until observed, were sputter coated with gold and observed with an S-360 scanning electron microscope (Leo, Cambridge, USA).

\section{Statistical Analysis}

All graphs were generated using GraphPad Prism 5 software (GraphPad Software). Means and standard deviations (SD) were calculated. Statistical analysis was carried out by twoway repeated-measures analysis of variance (ANOVA) with Bonferroni post hoc tests. Both tests were performed used using GraphPad software. Differences between samples were considered statistically different for $p$-values lower than 0.05 .

\section{Nucleotide Sequence Accession Numbers}

The complete genome sequences of the two $P$. mirabilis phage isolates were submitted to the GenBank under the accession numbers KP890822 (vB_PmiP_5460) and KP890823 (vB_PmiM_5461).

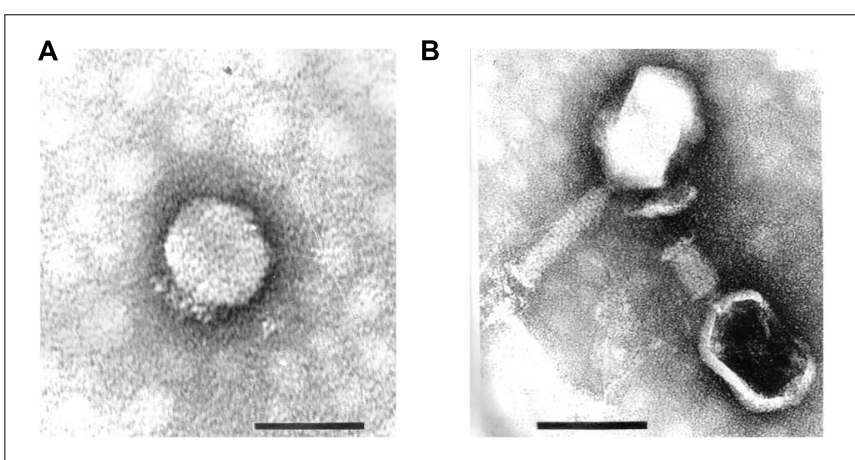

FIGURE 1 | Transmission electron micrographs of Proteus mirabilis phages: (A) Pm5460; (B) Pm5461. Scale bars represent $100 \mathrm{~nm}$. 


\section{RESULTS}

\section{Isolation and Morphology of Proteus mirabilis Phages}

These strains were used for phage enrichment (Table 1). In the initial screening, two different plaque morphotypes were detected. These plaques were purified and two different phages were isolated - vB_PmiP_5460 (5460) and vB_PmiM_5461 (5461), using P. mirabilis SGSC 5460 and SGSC 5461 as hosts, respectively. According to the morphological evaluation, phage 5460 belongs to the Podoviridae family of phages having a capsid $65 \mathrm{~nm}$ in diameter, a short $(13 \mathrm{~nm})$ tail terminating in tail fibers (Figure 1A). Phage 5461 has isometric head $87 \mathrm{~nm}$ in diameter, and a contractile tails ( $110 \mathrm{~nm}$ long and $17 \mathrm{~nm}$ wide) and belongs to the Myoviridae family (Figure 1B). Phage 5460 forms clear plaques $1.5 \mathrm{~mm}$ in diameter while phage 5461 forms smaller $(1 \mathrm{~mm})$ and less clear plaques.

\section{Host Range Screening}

To investigate the host specificity of both phages, a total of 43 strains of Enterobacteriaceae listed in Table 1 were used. Phage 5460 lysed 12 out of 18 P. mirabilis strains (67\%), three out of six $P$. vulgaris strains and one tested $P$. penneri strain; while phage 5461 killed all (100\%) of the Proteus spp. tested. Notwithstanding the fact that only 43 strains were tested, both phages seem to have a Proteus-genus-specific profile, having no activity against other Enterobacteriaceae strains. It is likely that phage 5461 recognizes a conserved outer membrane protein (OMP) or polysaccharide such as the inner core region of lipopolysaccharide (LPS) which is known to be conserved among at least $P$. mirabilis strains (Vinogradov and Perry, 2000; Kaca et al., 2011).

\section{One-step Growth Curve}

The replication of phages 5460 and 5461 on their respective hosts (Supplementary Figure S2) revealed a latent and rise periods of phage 5460 of approximately 10 and $15 \mathrm{~min}$, and an average burst size of 46 PFU per infected cell. On the other hand, phage 5461 showed a latency period of $25 \mathrm{~min}$, a rise period of $10 \mathrm{~min}$ and a burst size of 11 PFU per infected cell. Within the $60 \mathrm{~min}$ of duration of the experiment, a second cycle of replication was evident for both phages.

\section{Genome Analysis}

Genome analysis revealed that both phages are virulent, not encoding any genes associated with lysogeny. Furthermore, no known virulence-associated or toxic proteins were detected in silico, revealing that both phages are potentially safe for therapeutic purposes.

Phage 5460 has a linear double-stranded DNA with 44,573 bp with a $\mathrm{G}+\mathrm{C}$ content of $39.6 \%$ (Table 2). This phage encodes 53 putative CDSs, tightly packed occupying $93 \%$ of its genome. Of the CDSs, 20 have an assigned function and 11 are unique (Supplementary Table S1A). No tRNA genes were detected. The majority (90.6\%) of the CDSs possess methionine as start codon, while only $5 \%$ a GTG start codon. BLASTN searches revealed that 5460 is homologous to the Autographivirinae phages: K15 (Escherichia coli), UAB_Phi78 (Salmonella Typhimurium) and SP6 (Salmonella Typhimurium). The comparison of the nucleotides of 5460 and these three phages with EMBOSS stretcher showed that 5460 has identities around $60 \%$ with these phages, being most similar (63.4\%) to phage SP6 while CoreGenes (40) analysis showed that these phages share 33 homologs, which represent $62.3 \%$ of the 5460 proteins. Furthermore, the phylogenetic tree of the DNA polymerase containing homologs to 5460, demonstrated that this phage shares a branch with Proteus phages PM_85 and PM_93, being very close to the Enterobacteria phage SP6 (Supplementary Figure S3A). Consequently, this phage can be assigned to the Sp6virus genus.

Phage 5461 has a linear double-stranded DNA with 161,989 bp with a GC content of $31.1 \%$, codifying 256 putative CDSs that occupy $95.2 \%$ of the genome (Table 2). From these CDSs, 123 have assigned function, while 90 are unique having no significant homologies to any proteins on public databases (Supplementary Table S1B). Ninety-five percent of the CDSs have an ATG as a start codon, while TTG (3\%) and GTG (2\%) are also used by this phage. This phage encodes eight different tRNA genes (tRNA-Glu, tRNA-Ser, tRNA-Asp, tRNA-Gly, tRNA-Pro, tRNAMet, tRNA-Tyr, tRNA-Arg). BLASTN searches showed that the Yersinia phage phiR1-RT and Salmonella phages STP4-a and S16 are the closest relatives and therefore it can be assumed that this phage is part of the Tevenvirinae subfamily. EMBOSS stretcher alignment showed that 5461 and Yersinia phage phiR1RT have an identity of $53.1 \%$, while 5461 shares $52.2 \%$ of identity with Salmonella phages STP4-a and S16. CoreGenes (40) results showed that 5461 shares 133 homologous proteins with these three phages, which represent $45.3 \%$ of total proteins of 5461 . The phylogenetic tree comprising the homologs of 5461 terminase large subunit shows that 5461 along with Serratia phage PS2 and Citrobacter phage Merlin are between the two ICTV approved taxa - Js98virus and Cc31virus (Supplementary Figure S3B).

\section{Screening of $P$. mirabilis Strains for Biofilm Formation Ability}

Phage stability in AU was assessed and both phages were shown to keep their titer after incubation in AU for $168 \mathrm{~h}$ (data not shown).

Then, an initial screening was performed to select strong biofilm-forming P. mirabilis strains according to the classification reported by Stepanovic et al. (2000). In total, biofilms of 15 different $P$. mirabilis strains were formed in $\mathrm{AU}$ for $48 \mathrm{~h}$ and the total biofilm biomass quantified (Figure 2). The vast majority of the strains were classified as moderate biofilm forming, five were defined as weak biofilm forming strains, $P$. mirabilis CECT 4101 was the only strain classified as a non-biofilm forming strain, and $P$. mirabilis strains SGSC 5449 and SGSC 5446 were the only strong biofilm-forming strains.

\section{Evaluation of the Amount of Phages Coating the Silicone Surface}

An efficient phage coating of the surfaces is critical to assure the success of an anti-biofilm strategy. Physical adsorption has been 
TABLE 2 | Proteus mirabilis phages genome properties.

\begin{tabular}{|c|c|c|c|c|c|c|}
\hline Phage & Genome size (Kb) & $\mathrm{G}+\mathrm{C}$ content $(\mathrm{mol} \%)$ & Putative CDSs & Promoters/terminators & tRNAs & Closest homolog (\% identity) \\
\hline Pm5460 & 44,573 & 39.6 & 56 & $10 / 5$ & 0 & Enterobacteria phage SP6 (63.4) \\
\hline Pm5461 & 161,989 & 31.1 & 256 & $34 / 15$ & 8 & Yersinia phage phiR1-RT (53.1) \\
\hline
\end{tabular}

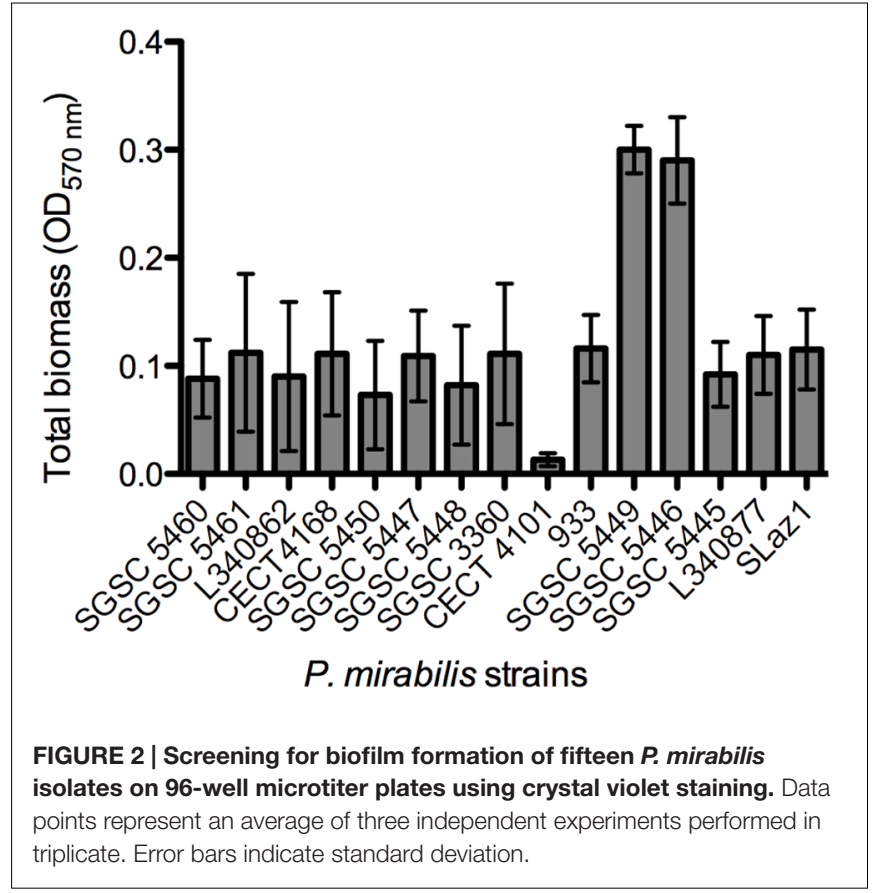

used to immobilize bacteriophages (Hosseinidoust et al., 2011), however, it cannot be assured a correct orientation of the phage (as tails must be available to interact with bacteria; Cademartiri et al., 2010). To better evaluate the effectiveness of phage physical adsorption, different phage concentrations were placed in contact with silicone surfaces (Figure 3). The amount of phages retained at the silicone surfaces increased proportionally to the initial concentration. The amount of 5460 phage particles adsorbed to the silicone surfaces was in average 1 log higher than the amount of 5461 phages (Figure 3).

\section{Evaluation of Phage Release from Silicone Surfaces}

The number of phages released from silicone surfaces was determined over time (Figure 4). The amount of phages 5460 released from the surfaces was in the range of $10^{3}$ PFU.mL ${ }^{-1}$ (around 1\% of the adhered phages). Phage 5461 was almost completely released from the surface after $2 \mathrm{~h}$ in contact with AU.

\section{Dynamic Model for Biofilm Formation}

The conditions found in real urinary catheters were mimicked using a continuous biofilm model system using Foley catheters. The viable cell counts and microscopy imaging of phage cocktailcoated catheters were compared with the control (non-phage coated) catheters (Figure 5). The higher concentration of biofilm cells obtained in each independent experiment was used to normalize the data.

During the assays, bacterial concentrations ranged between $\log 6$ and $\log 8$. According to the results, the phage-coating led to the reduction of the biofilm population and that difference was even more evident with the increase of biofilm age. Despite the non-significant differences of viable cells after $48 \mathrm{~h}$ between the

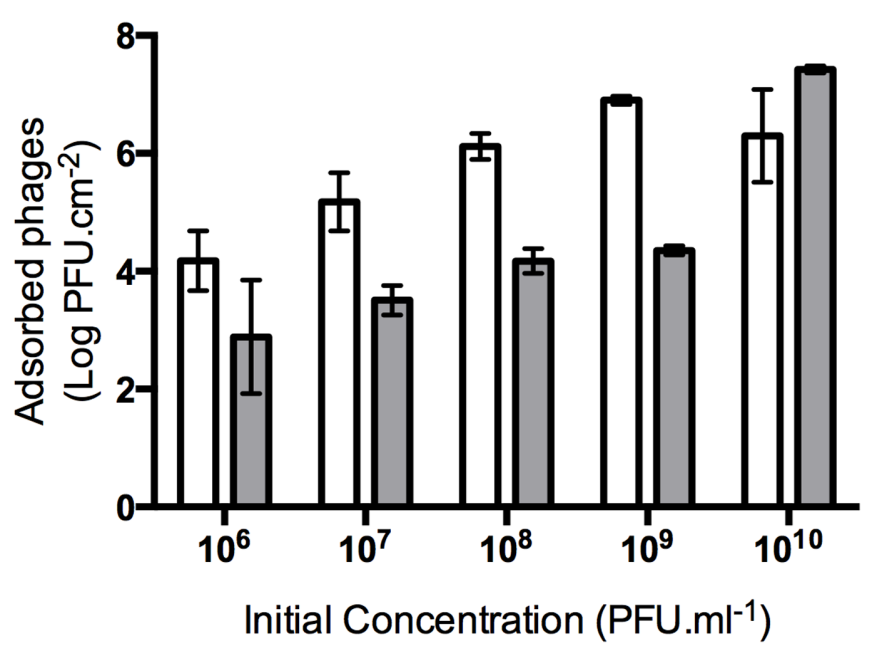

FIGURE 3 | Number of phages coating silicone surfaces per surface area. Data points represent an average of three independent experiments performed in triplicate and error bars indicate standard deviation. Statistical differences $(p<0.05)$ between the phage initial concentration and adsorbed phages were determined by two-way repeated-measures analysis of variance (ANOVA) with Bonferroni post hoc test. 


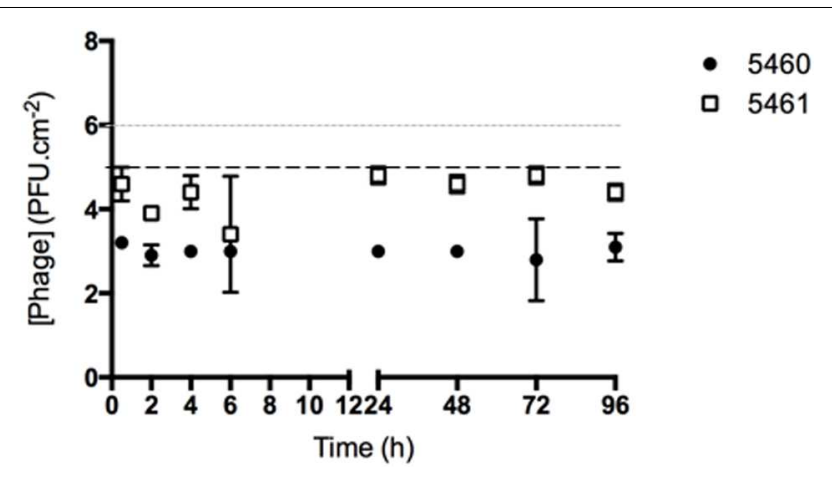

FIGURE 4 | Number of phages released from silicone surfaces per surface area. Data points represent an average of three independent experiments performed in triplicate and error bars indicate standard deviation. The approximate concentration of adhered 5460 phage is presented in the graphic as a black dot intermittent gray line, while approximate concentration of adhered 5461 phage is presented as an intermittent black line. Statistical differences $(p<0.05)$ between the approximate initial phage titer with released phage were determined by two-way repeated-measures analysis of variance (ANOVA) with Bonferroni post hoc test.

phage-coated or non-coated catheters sections, a clear tendency of the phage cocktail to reduce $P$. mirabilis biofilms was already observable (Figure 5) leading to significant reductions $(p<0.05)$ at 96 and $168 \mathrm{~h}$. This was also confirmed by epifluorescence and SEM (Figure 5) microscopy where more cells were observed in control catheter sections than in phage-coated catheters (Figure 5).

\section{DISCUSSION}

Urinary tract infections are involved in $40 \%$ of all nosocomial infections (Saint et al., 2008). Although Gram-positive bacteria, such as Staphylococcus epidermidis and Enterococcus faecalis can cause these infections, Gram-negative Enterobacteriaceae, are the most commonly implicated in CAUTI development (Siddiq and Darouiche, 2012). P. mirabilis forms crystalline biofilms within the urinary tract and is responsible for up to $30 \%$ of all urinary tract stones (struvite) (Stickler and Morgan, 2006). Furthermore, these crystalline structures recurrently block the flow through catheters (Stickler and Feneley, 2010). As frequently observed with different bacterial species, $P$. mirabilis strains are important reservoirs of antibiotic resistant determinants (Harada et al., 2014) and, because of this, resistant phenotypes have emerged in last years (Harada et al., 2014; Wang et al., 2014). Furthermore, as in other species, $P$. mirabilis biofilms were shown to be more tolerant to several antibiotics, than their planktonic counterparts (Moryl et al., 2013).

On the last decades the use of phages as alternatives or complements to antibiotic therapy has extensively been evaluated (Viertel et al., 2014) and has even been listed by the US National Institute of Allergy and Infectious Diseases as one important approach to combat antibiotic resistance (Reardon, 2014). The current relevance of phage therapy associated with the lack of
P. mirabilis phages described so far, were the underlying reasons for this study.

Two $P$. mirabilis phages, isolated from raw effluents of wastewater treatment plants, were characterized. TEM analysis demonstrated that both belong to the Caudovirales order and are, respectively, members of the Podoviridae (phage 5460) and the Myoviridae (phage 5461) families. Their spectrum of activity against a collection of Proteus spp. was 16 out of 26 strains for phage 5460 and 26 out of 26 strains for 5461 . Both phages were unable of killing other Enterobacteriaceae strains tested. Despite both phages have shown specificity to Proteus genera, it should be noted that only a limited number of strains of other species was used. As expected, these two phages had clear differences in the replication parameters, which resulted in higher burst size for the Podoviridae phage.

Based upon the genome sequence, phage 5460 belongs to the Autographivirinae subfamily, specifically to the Sp6virus genus. The GC content of phage 5460 is $39.6 \%$, a value similar to P. mirabilis HI4320 strain (GC 38.9\%) and to Proteus phage PM16 (41.4\%G+C) and 5460 shares protein homology with enterobacterial Podoviridae phages K1-5 (63.5\%), SP6 (63.5\%), and UAB_Phi78 (56.9\%).

Despite the morphological similarities with Myoviridae of the T4-like phages, phage 5461 has different characteristics from those deposited in GenBank. For instance, the GC content of 5461 is $31.1 \%$, which is clearly lower than the content observed in other T4-like viruses and in its host species. This low GC content may cause codon usage problems during phage infection (Rocha and Danchin, 2002). The presence of eight tRNA genes on 5461 genome might attenuate these differences, as the presence of tRNAs in phage genomes is correlated with the differences in the codon usage between the phage and the host, corresponding to codons that are expected to be poorly translated by the host machinery (Bailly-Bechet et al., 2007). Phage 5461 shares homologous proteins with the myoviruses Yersinia phage phiR1RT (44.3\%) and Salmonella phages STP4-a (45.1\%) and S16 (43.3\%). Furthermore, the phylogenetic analysis have shown that this phage cannot be assigned to a known genus.

The two phages characterized were studied for their potential preventive activity toward biofilms. For this, silicone surfaces were coated with phages using standard adsorption procedure. The fact that phage 5460 adsorbed in greater amounts than phage 5461 can be due to the different morphological characteristics and composition of the phages. Hosseinidoust et al. (2011), described that the morphology of the phages has a great influence on phage adsorption due to their orientation on the surface. Podoviruses are shorter that myoviruses, therefore the ratio between the surface area and the phage length is greater for podophages, consequently it is expected that a greater quantity of 5460 are adsorbed to silicone surfaces.

In order to obtain a stable coating it is important that phages remained attached to the surface during flow (Lehman and Donlan, 2015), therefore phage release from surfaces was also evaluated by titer determination. The results pointed out to a greater adhesion strength between phage 5460 and the silicone surface compared to phage 5461 . It has been previously shown 


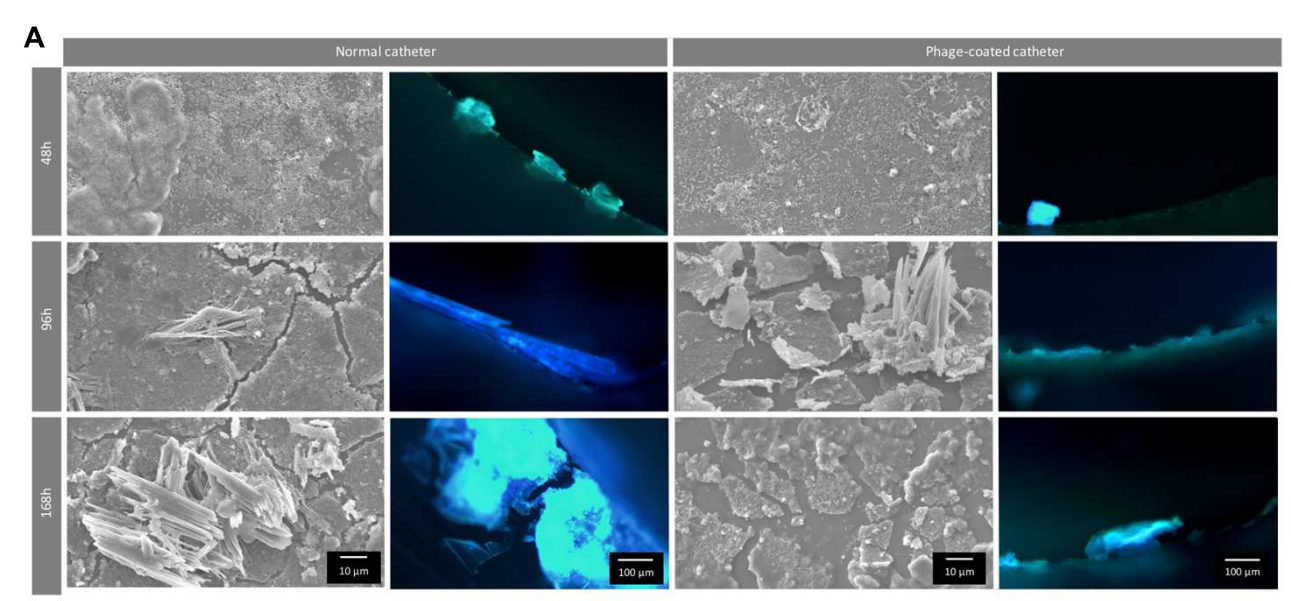

B

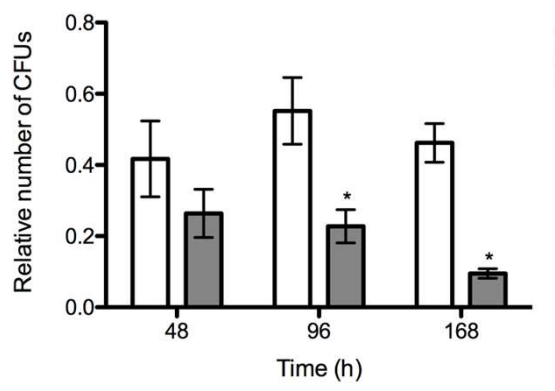

FIGURE 5 | Phage cocktail effect on $\boldsymbol{P}$. mirabilis biofilms under dynamic conditions. Representative SEM and epifluorescence images (A) and relative number of $\mathrm{CFU}(\mathbf{B})$ of $P$. mirabilis biofilms formed in normal and phage-coated Foley catheter show a decrease on the biofilm population, as well as on the overall biomass. SEM images are not representative of the overall biofilm as they are focused on spaces where biofilm is present. Relative CFU values were normalized using the higher concentration of biofilm cells obtained in each independent experiment. Magnification of 100x and 1000x, were used for epifluorescence and SEM images, respectively. Data points represent an average of four independent experiments and error bars indicate standard deviation. Statistical differences $(p<0.05)$ between control biofilms and phage-cocktail- -treated biofilms $\left(^{*}\right)$ were determined by two-way repeated-measures analysis of variance (ANOVA) with Bonferroni post hoc test.

that phages from different families have different surface-coating properties (Hosseinidoust et al., 2011). However, the reason why the coating stability differs between phages is not apparent as the phages major capsid proteins - the most abundant component of the phage particle - present similar isoelectric points (phage 5460: 5.15; phage 5461: 5.10) and hydrophibicities (phage 5460: -0.23 ; phage 5461: -0.33 ), which would suggest the same ability to interact with the surface. Although the majority of the adhered phages are released in short periods, the host can colonize the catheter surface forming biofilms. At this stage, not only the microbial cells adhered to the catheter surface, but also the biofilm by itself are phage reservoirs (Doolittle et al., 1996). Therefore, phages within the biofilm will be available to infect neighbor cells delaying biofilm formation and development.

After performing a rapid screening for biofilm formation in different $P$. mirabilis strains, two good biofilm-forming strains were selected for further tests in dynamic models. The two good biofilm-forming strains have shown similar susceptibly to both phages and thus have provided a suitable combination for the dynamic studies.
Due to their distinct lytic spectra and surface binding characteristics, a cocktail of both phages was used to prevent biofilm formation under dynamic conditions. Since urinary catheters can stay inserted in the patients' bladder for long periods, the efficacy of phage-coated catheters was prolonged up to 7 days. In fact, the phage cocktail developed herein reduced significantly the total number of cultivable cells after 96 and $168 \mathrm{~h}$.

An important feature of $P$. mirabilis biofilms is its great ability to form crystals, which associated with bacterial cells attached to surfaces can lead to clogging of the catheter (Coker et al., 2000; Stickler et al., 2006). During our experiments this ability was evident as crystalline biofilms were seen microscopically and even by simple observation of the tubing at naked eye. Indeed, the difference in biofilm biomass in phage-coated or non-coated catheters were also apparent macroscopically.

Other authors have evaluated the use of a phage cocktail in the prevention and eradication of E. coli and P. mirabilis biofilms (Carson et al., 2010) and observed significant reductions (3 to 4 $\log$ ) in the E. coli population, however, only a 1 log reduction was observed for the P. mirabilis population. These authors suggested 
that this lower efficacy might be related with phage-dependent factors, such as the production of depolymerases and the ability to penetrate the EPS matrix. In the case of the phage cocktail developed herein, this can also be one of the reasons for the low reductions $(1 \mathrm{log}$ ) observed for the $P$. mirabilis biofilm. More recently, Lehman and Donlan (2015) used a phage cocktail on the pre-treatment of hydrogen-coated silicone catheters to prevent the adhesion of Pseudomonas aeruginosa and $P$. mirabilis. After treatment, the authors detected a higher decrease on CFU counts of $P$. aeruginosa than of $P$. mirabilis. Nonetheless, reduction in the $P$. mirabilis population was still pronounced with a $\log$ reduction of approximately 2, for both single and mixed biofilms.

Other reasons that might be hampering phage efficacies might be physico-chemical factors such as $\mathrm{pH}$ and ions (Jonczyk et al., 2011) and also the fact that the adsorption of phages to their target cells may be inhibited by the crystals formed in AU during biofilm formation. Furthermore, the biofilm matrix itself can block the specific phage receptors, preventing phage infection from occurring (Samson et al., 2013). Finally, the physiological state of the cells could also be influencing phage efficacy, since log-phase cells are faster and more efficiently lysed than cells with lower metabolic rates, such as the ones that compose biofilms (Hadas et al., 1997).

The specificity of the phages, their genome features, which do not encode genes associated with lysogeny, together with the moderate reductions obtained using in vitro simulated conditions, support the use of both phages vB_PmiP_Pm5460 and vB_PmiM_Pm5461 for preventing P. mirabilis biofilm formation on silicone catheters.

\section{REFERENCES}

Ackermann, H. W. (2009). Basic phage electron microscopy. Methods Mol. Biol. 501, 113-126. doi: 10.1007/978-1-60327-164-6_12

Altschul, S. F., Gish, W., Miller, W., Myers, E. W., and Lipman, D. J. (1990). Basic local alignment search tool. J. Mol. Biol. 215, 403-410. doi: 10.1016/S00222836(05)80360-2

Altschul, S. F., Madden, T. L., Schaffer, A. A., Zhang, J., Zhang, Z., Miller, W., et al. (1997). Gapped BLAST and PSI-BLAST: a new generation of protein database search programs. Nucleic Acids Res. 25, 3389-3402. doi: 10.1093/nar/25.17.3389

Armbruster, C. E., and Mobley, H. L. (2012). Merging mythology and morphology: the multifaceted lifestyle of Proteus mirabilis. Nat. Rev. Microbiol. 10, 743-754. doi: $10.1038 /$ nrmicro 2890

Azeredo, J., and Sutherland, I. W. (2008). The use of phages for the removal of infectious biofilms. Curr. Pharm. Biotechnol. 9, 261-266. doi: $10.2174 / 138920108785161604$

Aziz, R. K., Bartels, D., Best, A. A., DeJongh, M., Disz, T., Edwards, R. A., et al. (2008). The RAST Server: rapid annotations using subsystems technology. BMC Genomics 9:75. doi: 10.1186/1471-2164-9-75

Bailey, T. L., Boden, M., Buske, F. A., Frith, M., Grant, C. E., Clementi, L., et al. (2009). MEME SUITE: tools for motif discovery and searching. Nucleic Acids Res. 37, W202-W208. doi: 10.1093/nar/gkp335

Bailly-Bechet, M., Vergassola, M., and Rocha, E. (2007). Causes for the intriguing presence of tRNAs in phages. Genome Res. 17, 1486-1495. doi: 10.1101/gr.6649807

Brooks, T., and Keevil, C. W. (1997). A simple artificial urine for the growth of urinary pathogens. Lett. Appl. Microbiol. 24, 203-206. doi: 10.1046/j.1472765X.1997.00378.x

Broomfield, R. J., Morgan, S. D., Khan, A., and Stickler, D. J. (2009). Crystalline bacterial biofilm formation on urinary catheters by urease-producing urinary

\section{AUTHOR CONTRIBUTIONS}

NC, CA, JA, and SS conceived the study. AK, CA, JA, and SS analyzed data. LM and PV performed experiments. LM wrote the paper. All authors read and approved the final manuscript.

\section{FUNDING}

This study was supported by the Portuguese Foundation for Science and Technology (FCT) under the scope of the strategic funding of UID/BIO/04469/2013 unit, COMPETE 2020 (POCI01-0145-FEDER-006684) and by the Portuguese Foundation for Science and Technology (FCT) under the scope of the Project RECI/BBB-EBI/0179/2012 (FCOMP-01-0124-FEDER027462). NC and SS also thank FCT for the individual support through Investigador FCT contracts.

\section{ACKNOWLEDGMENT}

The authors thank Dr. Hans W. Ackermann for the TEM imaging.

\section{SUPPLEMENTARY MATERIAL}

The Supplementary Material for this article can be found online at: http://journal.frontiersin.org/article/10.3389/fmicb. 2016.01024

tract pathogens: a simple method of control. J. Med. Microbiol. 58(Pt 10), 1367-1375. doi: 10.1099/jmm.0.012419-0

Burton, D. C., Edwards, J. R., Srinivasan, A., Fridkin, S. K., and Gould, C. V. (2011). Trends in catheter-associated urinary tract infections in adult intensive care units-United States, 1990-2007. Infect. Control Hosp. Epidemiol. 32, 748-756. doi: 10.1086/660872

Cademartiri, R., Anany, H., Gross, I., Bhayani, R., Griffiths, M., and Brook, M. A. (2010). Immobilization of bacteriophages on modified silica particles. Biomaterials 31, 1904-1910. doi: 10.1016/j.biomaterials.2009. 11.029

Carson, L., Gorman, S. P., and Gilmore, B. F. (2010). The use of lytic bacteriophages in the prevention and eradication of biofilms of Proteus mirabilis and Escherichia coli. FEMS Immunol. Med. Microbiol. 59, 447-455. doi: 10.1111/j.1574-695X.2010.00696.x

Chenoweth, C. E., Gould, C. V., and Saint, S. (2014). Diagnosis, management, and prevention of catheter-associated urinary tract infections. Infect. Dis. Clin. North Am. 28, 105-119. doi: 10.1016/j.idc.2013.09.002

Coker, C., Poore, C. A., Li, X., and Mobley, H. L. (2000). Pathogenesis of Proteus mirabilis urinary tract infection. Microbes Infect. 2, 1497-1505. doi: 10.1016/S1286-4579(00)01304-6

Dereeper, A., Guignon, V., Blanc, G., Audic, S., Buffet, S., Chevenet, F., et al. (2008). Phylogeny.fr: robust phylogenetic analysis for the non-specialist. Nucleic Acids Res. 36, W465-W469. doi: 10.1093/nar/gkn180

Doolittle, M. M., Cooney, J. J., and Caldwell, D. E. (1996). Tracing the interaction of bacteriophage with bacterial biofilms using fluorescent and chromogenic probes. J. Ind. Microbiol. 16, 331-341. doi: 10.1007/BF015 70111

Finn, R. D., Bateman, A., Clements, J., Coggill, P., Eberhardt, R. Y., Eddy, S. R., et al. (2014). Pfam: the protein families database. Nucleic Acids Res. 42, D222-D230. doi: $10.1093 /$ nar/gkt1223 
Getliffe, K., and Newton, T. (2006). Catheter-associated urinary tract infection in primary and community health care. Age Ageing 35, 477-481. doi: 10.1093/ageing/afl052

Gu, J., Liu, X., Li, Y., Han, W., Lei, L., Yang, Y., et al. (2012). A method for generation phage cocktail with great therapeutic potential. PLoS ONE 7:e31698. doi: 10.1371/journal.pone.0031698

Hadas, H., Einav, M., Fishov, I., and Zaritsky, A. (1997). Bacteriophage T4 development depends on the physiology of its host Escherichia coli. Microbiology 143(Pt 1), 179-185. doi: 10.1099/00221287-143-1-179

Harada, K., Niina, A., Shimizu, T., Mukai, Y., Kuwajima, K., Miyamoto, T., et al. (2014). Phenotypic and molecular characterization of antimicrobial resistance in Proteus mirabilis isolates from dogs. J. Med. Microbiol. 63(Pt 11), 1561-1567. doi: $10.1099 /$ jmm.0.081539-0

Hoeniger, J. F. M. (1965). Development of flagella by Proteus mirabilis. J. Gen. Microbiol. 40, 29-42. doi: 10.1099/00221287-40-1-29

Hosseinidoust, Z., Van de Ven, T. G., and Tufenkji, N. (2011). Bacterial capture efficiency and antimicrobial activity of phage-functionalized model surfaces. Langmuir 27, 5472-5480. doi: 10.1021/la200102z

Jacobsen, S. M., Stickler, D. J., Mobley, H. L. T., and Shirtliff, M. E. (2008). Complicated catheter-associated urinary tract infections due to Escherichia coli and Proteus mirabilis. Clin. Microbiol. Rev. 21, 26-59. doi: 10.1128/cmr.00 019-07

Jonczyk, E., Klak, M., Miedzybrodzki, R., and Gorski, A. (2011). The influence of external factors on bacteriophages-review. Folia Microbiol. (Praha) 56, 191-200. doi: 10.1007/s12223-011-0039-8

Jones, B. V., Mahenthiralingam, E., Sabbuba, N. A., and Stickler, D. J. (2005). Role of swarming in the formation of crystalline Proteus mirabilis biofilms on urinary catheters. J. Med. Microbiol. 54(Pt 9), 807-813. doi: 10.1099/jmm.0.46123-0

Kaca, W., Glenska, J., Lechowicz, L., Grabowski, S., Brauner, A., and Kwinkowski, M. (2011). Serotyping of Proteus mirabilis clinical strains based on lipopolysaccharide O-polysaccharide and core oligosaccharide structures. Biochemistry (Mosc.) 76, 851-861. doi: 10.1134/S0006297911070169

Kall, L., Krogh, A., and Sonnhammer, E. L. (2004). A combined transmembrane topology and signal peptide prediction method. J. Mol. Biol. 338, 1027-1036. doi: 10.1016/j.jmb.2004.03.016

Kall, L., and Sonnhammer, E. L. (2002). Reliability of transmembrane predictions in whole-genome data. FEBS Lett. 532, 415-418. doi: 10.1016/S00145793(02)03730-4

Kropinski, A. M., Mazzocco, A., Waddell, T. E., Lingohr, E., and Johnson, R. P. (2009). Enumeration of bacteriophages by double agar overlay plaque assay. Methods Mol. Biol. 501, 69-76. doi: 10.1007/978-1-60327-164-6_7

Laslett, D., and Canback, B. (2004). ARAGORN, a program to detect tRNA genes and tmRNA genes in nucleotide sequences. Nucleic Acids Res. 32, 11-16. doi: 10.1093/nar/gkh152

Lavigne, R., Sun, W. D., and Volckaert, G. (2004). PHIRE, a deterministic approach to reveal regulatory elements in bacteriophage genomes. Bioinformatics 20, 629-635. doi: 10.1093/bioinformatics/btg456

Lehman, S. M., and Donlan, R. M. (2015). Bacteriophage-mediated control of a two-species biofilm formed by microorganisms causing catheter-associated urinary tract infections in an in vitro urinary catheter model. Antimicrob. Agents Chemother. 59, 1127-1137. doi: 10.1128/AAC.03786-14

Levering, V., Wang, Q., Shivapooja, P., Zhao, X., and Lopez, G. P. (2014). Soft robotic concepts in catheter design: an on-demand fouling-release urinary catheter. Adv. Healthc. Mater. 3, 1588-1596. doi: 10.1002/adhm.20140 0035

Melo, L. D., Sillankorva, S., Ackermann, H. W., Kropinski, A. M., Azeredo, J., and Cerca, N. (2014a). Characterization of Staphylococcus epidermidis phage vB_SepS_SEP9 - a unique member of the Siphoviridae family. Res. Microbiol. 165, 679-685. doi: 10.1016/j.resmic.2014.09.012

Melo, L. D., Sillankorva, S., Ackermann, H. W., Kropinski, A. M., Azeredo, J., and Cerca, N. (2014b). Isolation and characterization of a new Staphylococcus epidermidis broad-spectrum bacteriophage. J. Gen. Virol. 95(Pt 2), 506-515. doi: 10.1099/vir.0.060590-0

Morris, N. S., Stickler, D. J., and McLean, R. J. (1999). The development of bacterial biofilms on indwelling urethral catheters. World J. Urol. 17, 345-350. doi: $10.1007 / \mathrm{s} 003450050159$

Moryl, M., Torzewska, A., Jalmuzna, P., and Rozalski, A. (2013). Analysis of Proteus mirabilis distribution in multi-species biofilms on urinary catheters and determination of bacteria resistance to antimicrobial agents. Pol. J. Microbiol. $62,377-384$.

Naville, M., Ghuillot-Gaudeffroy, A., Marchais, A., and Gautheret, D. (2011). ARNold: a web tool for the prediction of Rho-independent transcription terminators. RNA Biol. 8, 11-13. doi: 10.4161/rna.8.1.13346

O'Hara, C. M., Brenner, F. W., and Miller, J. M. (2000). Classification, identification, and clinical significance of Proteus, Providencia, and Morganella. Clin. Microbiol. Rev. 13, 534-546. doi: 10.1128/CMR.13.4.534-546.2000

Pires, D., Sillankorva, S., Faustino, A., and Azeredo, J. (2011). Use of newly isolated phages for control of Pseudomonas aeruginosa PAO1 and ATCC 10145 biofilms. Res. Microbiol. 162, 798-806. doi: 10.1016/j.resmic.2011.06.010

Rahman, M., Kim, S., Kim, S. M., Seol, S. Y., and Kim, J. (2011). Characterization of induced Staphylococcus aureus bacteriophage SAP-26 and its anti-biofilm activity with rifampicin. Biofouling 27, 1087-1093. doi: 10.1080/08927014.2011.631169

Reardon, S. (2014). Phage therapy gets revitalized. Nature 510, 15-16. doi: $10.1038 / 510015 \mathrm{a}$

Rice, P., Longden, I., and Bleasby, A. (2000). EMBOSS: the European molecular biology open software suite. Trends Genet. 16, 276-277. doi: 10.1016/S01689525(00)02024-2

Rocha, E. P., and Danchin, A. (2002). Base composition bias might result from competition for metabolic resources. Trends Genet. 18, 291-294. doi: 10.1016/S0168-9525(02)02690-2

Saint, S., Kowalski, C. P., Kaufman, S. R., Hofer, T. P., Kauffman, C. A., Olmsted, R. N., et al. (2008). Preventing hospital-acquired urinary tract infection in the United States: a national study. Clin. Infect. Dis. 46, 243-250. doi: $10.1086 / 524662$

Saint, S., and Lipsky, B. A. (1999). Preventing catheter-related bacteriuria: should we? Can we? How? Arch. Intern. Med. 159, 800-808. doi: 10.1001/archinte.159.8.800

Sambrook, J., and Russell, D. W. (2001). Molecular Cloning: A Laboratory Manual, 3rd Edn. New York, NY: Cold Spring Harbor Laboratory Press.

Samson, J. E., Magadan, A. H., Sabri, M., and Moineau, S. (2013). Revenge of the phages: defeating bacterial defences. Nat. Rev. Microbiol. 11, 675-687. doi: 10.1038/nrmicro3096

Schattner, P., Brooks, A. N., and Lowe, T. M. (2005). The tRNAscan-SE, snoscan and snoGPS web servers for the detection of tRNAs and snoRNAs. Nucleic Acids Res. 33, W686-W689. doi: 10.1093/nar/gki366

Siddiq, D. M., and Darouiche, R. O. (2012). New strategies to prevent catheter-associated urinary tract infections. Nat. Rev. Urol. 9, 305-314. doi: 10.1038/nrurol.2012.68

Silva, S., Negri, M., Henriques, M., Oliveira, R., Williams, D., and Azeredo, J. (2010). Silicone colonization by non-Candida albicans Candida species in the presence of urine. J. Med. Microbiol. 59(Pt 7), 747-754. doi: 10.1099/jmm.0.017517-0

Stepanovic, S., Vukovic, D., Dakic, I., Savic, B., and Svabic-Vlahovic, M. (2000). A modified microtiter-plate test for quantification of staphylococcal biofilm formation. J. Microbiol. Methods 40, 175-179. doi: 10.1016/S01677012(00)00122-6

Stickler, D., Morris, N., Moreno, M. C., and Sabbuba, N. (1998). Studies on the formation of crystalline bacterial biofilms on urethral catheters. Eur. J. Clin. Microbiol. Infect. Dis. 17, 649-652. doi: 10.1007/s100960050150

Stickler, D. J., and Feneley, R. C. (2010). The encrustation and blockage of longterm indwelling bladder catheters: a way forward in prevention and control. Spinal Cord 48, 784-790. doi: 10.1038/sc.2010.32

Stickler, D. J., Lear, J. C., Morris, N. S., Macleod, S. M., Downer, A., Cadd, D. H., et al. (2006). Observations on the adherence of Proteus mirabilis onto polymer surfaces. J. Appl. Microbiol. 100, 1028-1033. doi: 10.1111/j.13652672.2006.02840.x

Stickler, D. J., and Morgan, S. D. (2006). Modulation of crystalline Proteus mirabilis biofilm development on urinary catheters. J. Med. Microbiol. 55(Pt 5), 489-494. doi: 10.1099/jmm.0.46404-0

Viertel, T. M., Ritter, K., and Horz, H.-P. (2014). Viruses versus bacteria-novel approaches to phage therapy as a tool against multidrug-resistant pathogens. J. Antimicrob. Chemother. 69, 2326-2336. doi: 10.1093/jac/dku173

Vinogradov, E., and Perry, M. B. (2000). Structural analysis of the core region of lipopolysaccharides from Proteus mirabilis serotypes O6, O48 and O57. Eur. J. Biochem. 267, 2439-2446. doi: 10.1046/j.1432-1327.2000.01262.x 
Wang, J. T., Chen, P. C., Chang, S. C., Shiau, Y. R., Wang, H. Y., Lai, J. F., et al. (2014). Antimicrobial susceptibilities of Proteus mirabilis: a longitudinal nationwide study from the Taiwan surveillance of antimicrobial resistance (TSAR) program. BMC Infect. Dis. 14:486. doi: 10.1186/1471-2334-14-486

Weber, D. J., Sickbert-Bennett, E. E., Gould, C. V., Brown, V. M., Huslage, K., and Rutala, W. A. (2011). Incidence of catheter-associated and non-catheterassociated urinary tract infections in a healthcare system. Infect. Control Hosp. Epidemiol. 32, 822-823. doi: 10.1086/661107

Wilkins, M. R., Gasteiger, E., Bairoch, A., Sanchez, J. C., Williams, K. L., Appel, R. D., et al. (1999). Protein identification and analysis tools in the ExPASy server. Methods Mol. Biol. 112, 531-552.

Zafar, N., Mazumder, R., and Seto, D. (2002). CoreGenes: a computational tool for identifying and cataloging "core" genes in a set of small genomes. BMC Bioinformatics 3:12. doi: 10.1186/1471-2105-3-12
Zuker, M. (2003). Mfold web server for nucleic acid folding and hybridization prediction. Nucleic Acids Res. 31, 3406-3415. doi: 10.1093/nar/gkg595

Conflict of Interest Statement: The authors declare that the research was conducted in the absence of any commercial or financial relationships that could be construed as a potential conflict of interest.

Copyright (c) 2016 Melo, Veiga, Cerca, Kropinski, Almeida, Azeredo and Sillankorva. This is an open-access article distributed under the terms of the Creative Commons Attribution License (CC BY). The use, distribution or reproduction in other forums is permitted, provided the original author(s) or licensor are credited and that the original publication in this journal is cited, in accordance with accepted academic practice. No use, distribution or reproduction is permitted which does not comply with these terms. 